\title{
Adjustment of the Exploration Grids and its use to increase the Reliability of Geological Models of Coal Deposits
}

\author{
Tamara Rogova ${ }^{1, *}$, and Sergey Shaklein ${ }^{2,3}$ \\ ${ }^{1}$ T.F. Gorbachev Kuzbass State Technical University, 650000, 28 Vesennyaya St., Kemerovo, Russia \\ ${ }^{2}$ Kemerovo branch of the Institute of Computational Technologies of Siberian Branch of the Russian \\ Academy of Sciences, 650025, 21 Rukavishnikova St., Kemerovo, Russia \\ ${ }^{3}$ Federal State Budget Scientific Institution «The Federal Research Center of Coal and Coal \\ Chemistry of Siberian Branch of the Russian Academy of Sciences», 650065, 10 Leningradskiy Ave., \\ Kemerovo, Russia
}

\begin{abstract}
The current procedure for determining the boundaries of geological domains, the allocation of which is the mandatory element of digital geological modelling, does not entirely take into account the specifics of coal deposits. Without its improvement, it is impossible to increase the reliability of geological models used in the implementation of the "Industry 4.0" strategy. A new method for analysis of geological data is supposed - the adjustment of the exploration grids method. It is to determine the corrections for values of measured parameters, the use of which eliminates the uncertainty of geological data interpretation. The correction values determined by the method of conditional measurements, which used at equalization geodetic networks. Corrections are considered as an indicator of the significance of measurement and interpolation errors which occurs in the vicinity of specific measurement points. The measured values of parameters are not corrected. Geological domains are the areas with close in values corrections, whose boundaries are corrections isolines. Separate single corrections of anomalous magnitude indicate the presence of extreme values parameters.
\end{abstract}

\section{Introduction}

The estimate of mineral resources and reserves is an essential task for mining engineers. The geological models of deposits built during its implementation used as a basis for detailed mine designing and planning [1]. Therefore, the quality of models directly affects the quality of mining projects.

The role of geological modelling is significantly increasing in connection with the development of the mining industry digitalization. Without reliable geological models, it is impossible to implement innovative technologies that meet the requirements of the "Industry 4.0" industrial strategy [2]. The implementation of this strategy is envisaged by the Program of the development of the Russian coal industry for the period until the 2035 year, which considers the creation of the "Intellectual Quarry" and the "Intellectual Mine"

\footnotetext{
* Corresponding author: rogovatb@kuzstu.ru
} 
complexes as the priority for the development of coal mining technologies.

Kriging methods are mainly used [3] for the development of geological models for various indicators of coal deposits (gypsometry and thickness of the seam, ash content, etc.). Based on the features of the geostatistics mathematical apparatus, the studied parameters within the model must satisfy the stationarity requirements, i.e. they must be uniform in the parameter distribution [4]. The uniformity is achieved by dividing the deposit into domains, i.e. into fragments which are uniform in geological terms and confidence level of the geological study. The uniformity of the domains established by statistical and geostatistical analysis, including the construction of histograms, the estimation of statistical parameters, the construction of variograms in orthogonal directions, the estimation of anisotropy, etc. [5].

Domaining within the conditions of coal deposits is complicated by the variable anisotropy of most of their properties and a small number of geological measurements within the individual domains. Obtaining the confidential estimates of the statistical parameters of the distributions and variograms of indicators in such conditions is impossible. It is recognized that in the conditions of coal deposits "sole use of the variogram is risky" [6].

Consequently, the methodology improvement for the domaining of coal deposits, the quality of the allocation of which is a condition for obtaining confident models of deposits, is an essential task. Its solution is possible based on a new method for the analysis of geological data suggested by the authors - the method of adjustment of the exploration grids.

\section{The proposed method}

Adjustment has been used in geodesy since the time of K. Gauss. Its necessity arose because, the measurement errors lead their results to become geometrically inconsistent with each other (for example, the sum of the measured angles in a triangle by the results of their measurements may differ from $180^{\circ}$ ). The value of inconsistency is usually called error of closure. To obtain consistent results of geodetic measurements, one should perform the mathematical process called adjustment [7].

In exploration practice, errors of closure do not occur. However, they can be obtained with artificially created conditions that carry quantitative information on the confidence of the interpretation of geological data.

Let's consider an exploration grid. We divide this grid into a system of cells - convex quadrangles with exploration wells as vertices (Fig. 1).

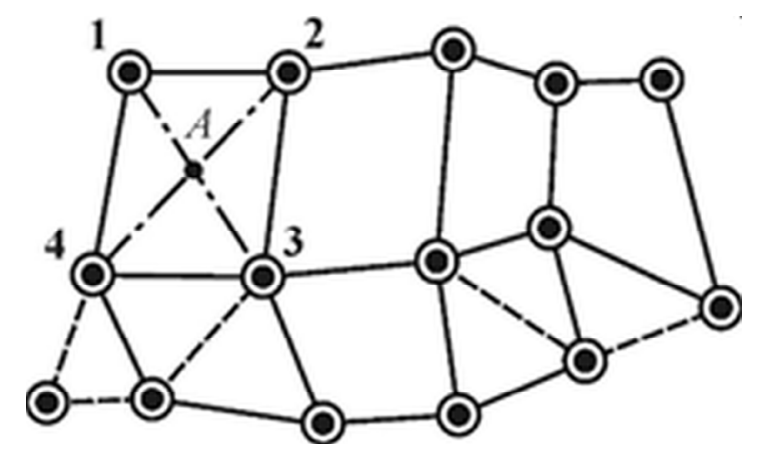

Fig. 1. Quadrangular cells of the exploration grid. 
The vertices of each quadrangle numbered with numbers from 1 to 4 , starting from an arbitrary vertex with the number increase in the clockwise direction. In each quadrangle, we draw two diagonals. For a 1-2-3-4 quadrilateral (Fig. 1), these are diagonals 1-3 and 2-4 intersecting at the point $\mathrm{A}$.

Using an interpolation method, one can determine the value of the parameter $P$ at the point $A$ from each diagonal $\left(P_{1-3}\right.$ and $\left.P_{2-4}\right)$. It is clear that theoretically $P_{1-3}=P_{2-4}$. However, due to measurement and interpolation errors, the values $P_{1-3}$ and $P_{2-4}$ will not coincide with each other. Therefore, their difference $D$, being the difference of two independent indirect definitions, can be considered as a numerical measure of the ambiguity of geological data interpretation, i.e., as the exploration criterion of the parameter study in the contour of the quadrangular cells of the exploration grid. Previously, this difference was successfully used to quantify the confidence of coal resource and their classification $[8,9]$.

Based on the theoretical equality of criterion $D$ to zero, its value can be considered as a kind of error of closure, that is, as a measure of fulfilling a geometric, objectively existing condition. Having the values of the criteria (error of closure) for all of the quadrangles of the grid, one can set the problem of adjustment: find the values (corrections) by which the measured values of the parameter should be changed so that the errors of closure for all the quadrangles of the exploratory grid would become equal to zero at the same time. Since the criteria $D$ are determined by measurement and interpolation errors, the corrections obtained objectively depend on the same factors. This dependence underlies adjustment as a method of analysis.

The methodology of the use of the adjustment results does not imply any changes in the results of measurements. Corrections are considered only as a measure of the significance of measurement and interpolation errors occurring in the measurement points vicinity.

It is recommended to perform the adjustment of the exploration grids by the method of conditional measurements, which ensures the corrections are under the minimum condition of the sum of their squares.

For example, during the adjustment of the thickness of seam or coal quality parameters, the criterion $D$ for quadrangle number $j$ is calculated using linear interpolation using the formula:

$$
D_{j}=\left|\left(P_{3 j}-P_{1 j}\right) x_{j}+P_{1 j}-\left(P_{4 j}-P_{2 j}\right) x_{j}^{\prime}-P_{2 j}\right|,
$$

where $x_{j}, x_{j}^{\prime}$ - the ratio of the length of the distance from the vertex 1 to the point $\mathrm{A}$ to the length of the diagonal 1-3 and the ratio of the length of the distance from the vertex 2 to the point A to the length of the diagonal 2-4 respectively (Fig. 1).

Let's consider $t$ quadrangles formed by $n$ prospecting bores, for each of which an error of closure is known - criterion $D_{j}$. To prepare the data for adjustment, Table 1 is formed, the rows of which correspond to the quadrangles, and the columns correspond to the prospecting bores.

Each cell of the table is filled with the values of the coefficients $a_{i j}(i$-serial number of the bores, $j$ - serial number of the quadrangle). If the bore $i$ is not included in quadrangle $j$, then $a_{i j}=0$, or if it is, then $a_{i j}$ is determined depending on the index number of the bore in the quadrangle (the vertex number of the quadrangle):

$$
\begin{array}{ll}
\text { - for vertex \# 1: } & a_{i j}=1-x ; \\
\text { - for vertex \# 2: } & a_{i j}=x^{\prime}-1 ; \\
\text { - for vertex \# 3: } & a_{i j}=x ; \\
\text { - for vertex \# 4: } & a_{i j}=-x^{\prime} .
\end{array}
$$


Thus, in each row of Table 1, there will be four nonzero numbers giving zero in their algebraic sum.

Table 1. Table of derivatives $a_{i j}$.

\begin{tabular}{|c|c|c|c|c|c|}
\hline $\begin{array}{c}\text { Sequence number of the } \\
\text { quadrangle }(\boldsymbol{k})\end{array}$ & \multicolumn{5}{|c|}{ Sequence number of the bores $(\boldsymbol{i})$} \\
\cline { 2 - 6 } & 1 & 2 & 3 & $\ldots$ & $n$ \\
\hline 1 & $a_{11}$ & $a_{21}$ & $a_{31}$ & $\ldots$ & $a_{n 1}$ \\
\hline 2 & $a_{12}$ & $a_{22}$ & $a_{32}$ & $\ldots$ & $a_{n 2}$ \\
\hline$\ldots$ & $\ldots$ & $\ldots$ & $\ldots$ & $\ldots$ & $\ldots$ \\
\hline$t$ & $a_{1 t}$ & $a_{2 t}$ & $a_{3 t}$ & $\ldots$ & $a_{n t}$ \\
\hline
\end{tabular}

Table 2 is formed with the sums of the products of the rows of Table 1 . So $S_{11}$ is the result of multiplying row 1 by itself:

$$
S_{11}=\sum_{i=1}^{t} a_{i 1}^{2} ; \quad S_{12}=\sum_{i=1}^{t}\left(a_{i 1} a_{i 2}\right) \text { etc. }
$$

Table 2. Table of the multiplication of Table 1 rows.

\begin{tabular}{|c|c|c|c|c|c|}
\hline $\begin{array}{c}\text { Line numbers from Table 1 } \\
(\boldsymbol{k})\end{array}$ & $\mathbf{1}$ & $\mathbf{2}$ & $\mathbf{3}$ & $\ldots$ & $\boldsymbol{t}$ \\
\hline 1 & $S_{11}$ & $S_{21}$ & $S_{31}$ & $\ldots$ & $S_{t 1}$ \\
\hline 2 & $S_{12}$ & $S_{22}$ & $S_{32}$ & $\ldots$ & $S_{t 2}$ \\
\hline$\ldots$ & $\ldots$ & $\ldots$ & $\ldots$ & $\ldots$ & $\ldots$ \\
\hline$t$ & $S_{1 t}$ & $S_{2 t}$ & $S_{3 t}$ & $\ldots$ & $S_{t t}$ \\
\hline
\end{tabular}

The data from table 2 gives a system of linear equations:

$$
\begin{aligned}
& S_{11} K_{1}+S_{21} K_{2}+\ldots+S_{t 1} K_{t}=-D_{1} \\
& S_{12} K_{1}+S_{22} K_{2}+\ldots+S_{t 2} K_{t}=-D_{2} \\
& \ldots \ldots \ldots \ldots \ldots \ldots \ldots \ldots \ldots \ldots \\
& S_{1 t} K_{1}+S_{2 t} K_{2}+\ldots+S_{t t} K_{t}=-D_{t}
\end{aligned}
$$

as a result of the solution of which, $K_{k}$ correlates are found.

After that, each $k$-th row of Table 1 is multiplied by the correlate $K_{k}$, and the resulting products are summed by columns:

$$
\varepsilon_{i}=\sum_{k=1}^{t} a_{i k} \cdot K_{k}
$$

The sums $\varepsilon_{i}$ are corrections to the parameters measured in $i$-th prospecting bores. From the algorithm above it follows that the algebraic sum of all corrections is equal to zero. The quality control of adjustment is provided by the repeated calculation of the criteria $D_{j}$ according to the parameter values changed by the values of the corrections. With high quality adjustment, the corrections should be equal to zero.

During the adjustment of elevations above sea level of the interception depths of coal seam, the criterion of exploration is applied, based on cubic spline interpolation, which 
takes into account the dip angles seam [8]. In this case, the specified sequence of adjustment remains the same when determining the coefficients $a_{i j}$ using the formulas:

$$
\begin{array}{ll}
\text { - for vertex \# 1: } & a_{i j}=1-(3-2 x) x^{2} ; \\
\text { - for vertex \# 2: } & a_{i j}=\left(3-2 x^{\prime}\right) x^{\prime 2}-1 ; \\
\text { - for vertex \# 3: } & a_{i j}=(3-2 x) x^{2} ; \\
\text { - for vertex \# 4: } & a_{i j}=-\left(3-2 x^{\prime}\right) x^{\prime 2} .
\end{array}
$$

Poor conditionality of the system of equations (3), which in practice can include up to 300 equations, makes it impossible to solve it using the Gaussian method which traditionally used in geodetic adjustment. However, its qualitative solution is always achieved with the use of iterative Trushnikov's method [10].

\section{Results and discussion}

The corrections obtained as a result of adjustment are the function of measurement and interpolation errors. Moreover, the interpolation errors depend on the configuration and density of the exploration grid and the complexity of the geological structure of the studied deposit. It follows that, within the same domain, the corrections do not undergo significant changes. Thus, the domaining is reduced to the allocation of areas with close values corrections.

When interpreting the results of adjustment of the elevations above sea level of the interception depths of coal seam, the sign of corrections is in great importance. In practice, there is often an alternation of a significant area with plus and minus signs of the corrections. These areas are domains with uniform hypsometry of seam. Their boundaries are isolines of zero-values corrections (Fig. 2, left). Such areas, as a rule, are located between tectonic disturbances, including those not yet known. In the absence of the need to allocate domains, corrections with plus and minus signs are placed chaotically and have small sizes.

When allocating domains by the thickness of seam or by coal quality parameters, it is necessary to focus not only on the sign but also on the correction values. A chaotic change in the values of the corrections indicates the absence of the need of domaining. (Fig. 2, right). Domains are allocated in the presence of significant areas with close values of corrections. Their boundaries are isolines of corrections, the vertical interval of which corresponds to a given interval of changes in corrections $\Delta$ in the domain. 

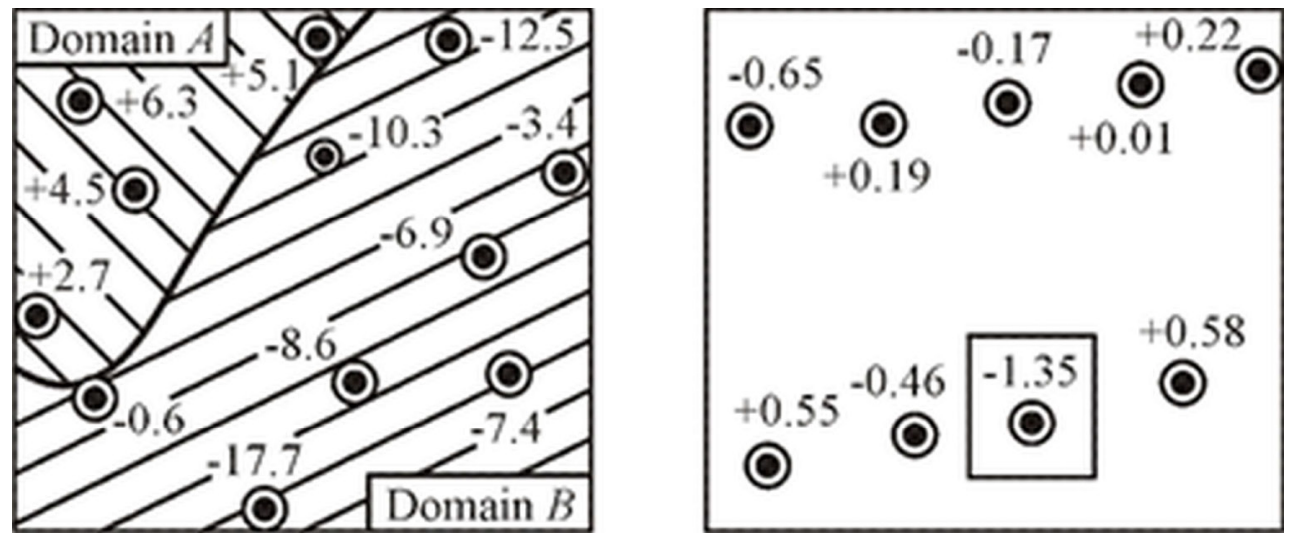

Fig. 2. Fragments of plans with the results of adjustment of the elevations above sea level of the interception depths of coal seam "Polysaevskiy II" (left) and thickness of seam "74" (right).

In the absence of any geological considerations for the choice of $\Delta$, it can be established by preliminary consideration of its possible values. Each $\Delta$ corresponds to a certain number of distinguished domains $N$. From the graph of the dependence of the number of domains $N$ on $\Delta$, it is possible to determine the optimal value $\Delta$ corresponding to the maximum number of domains (Fig. 3).

The construction of isolines - domain boundaries can be carried out by kriging methods. Their application is always correct - corrections always satisfy the requirement of stationarity; they distribute normally with the expected value equal to zero.

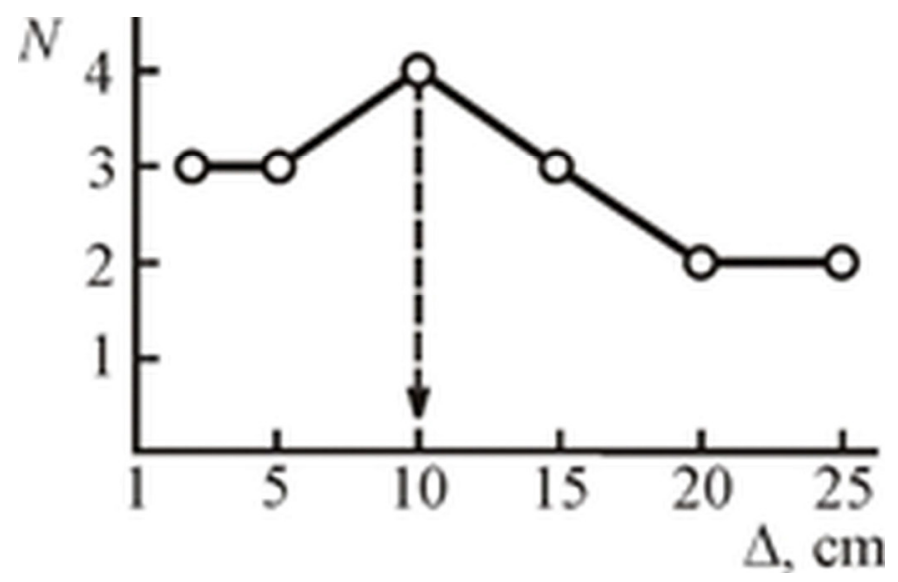

Fig. 3. The dependence of the number of domains $N$ the thickness of waste rock layers coal seam " 5 " on the accepted level of changes in the corrections $\Delta$ in the domain.

Before starting domaining extreme values of the parameter are restricted. The adjustment method provides the correct solution to this problem. If the correction value stands out sharply in its magnitude (for example, the correction " $-1.35 \mathrm{~m}$ " for the formation thickness highlighted in Fig. 2 (right)), it must be concluded that the parameter was determined in the bore with a significant error or in the bore vicinity there is a dramatic change in the behavior of the studied parameter. This method of identifying extreme parameter values automatically takes into account not only the result of the measurement but also its spatial position. Moreover, the value of each correction is determined 
simultaneously by all values of the parameter and the position of all bores throughout the considered area. Taking into account the normal distribution of corrections, it is not difficult to determine the critical level of corrections corresponding to the necessary probability of assessment. The practice of evaluating the Kuzbass coal deposits indicates the advisability of accepting the probability of occurrence of extreme grade measurements at the level of $7 \%$.

\section{Conclusion}

The proposed new method for the analysis of exploration data - the method of adjustment the exploration grids allows to formalize the procedures for allocating geological domains and extreme parameter values. The method has been successfully tested in the conditions of the Kuzbass coal deposits. The possibilities inherent in the method make it possible to use it later for the prediction of faulting and in geometrizing geological parameters.

\section{References}

1. S. K. Haldar, Mineral exploration: principles and applications (Elsevier, Oxford, 2018)

2. M. N. Sishi, A. Telukdarie, IEEE International Conference on Industrial Engineering and Engineering Management, 1, 201-205 (2017)

3. M. E. Rossi, C. V. Deutsch, Mineral Resource Estimation (Springer, New York, 2013)

4. T. Hengl, A Practical Guide to Geostatistical Mapping (University of Amsterdam, 2009)

5. Y. Z. Ma, Quantitative Geosciences: Data Analytics, Geostatistics, Reservoir Characterization and Modeling (Springer, Cham, 2019)

6. Australian Guidelines for the Estimation and Classification of Coal Resources: Guidelines Review Committee (AMEA, Adelaide, 2014)

7. H. Fan, Theory of Errors and Least Squares Adjustment (Royal Institute of Technology, Stockholm, 2010)

8. A. B. Efremenkov, T. B. Rogova, S. V. Shaklein, V. A. Efremenkov, IOP Conf. Series: Materials Science and Engineering, 656, 012016 (2019)

9. A. Kopytov, T. Rogova, S. Shaklein, Advances in Engineering Research, 176, 322-326 (2018)

10. V. N. Trushnikov, Computational Mathematics and Mathematical Physics, 22, 1, 239244 (1982) 